\title{
Les Associations et le Marketing : Une étude Exploratoire au Niveau des Associations Marocaines de la Province de Ouarzazate
}

\author{
El Kharraz, Ouail, PH
}

Faculté des Sciences Juridiques, Economiques et Sociales - Tanger de l'Université Abdelmalek Essaâdi, Maroc

Cherradi Laila, $P A$

Faculté Polydisciplinaire - Ouarzazate de l’Université Ibn Zohr, Maroc

Doi:10.19044/esj.2019.v15n16p247 URL:http://dx.doi.org/10.19044/esj.2019.v15n16p247

\section{Résumé}

Le marketing constitue aujourd'hui un facteur clé pour la réussite de toute organisation. Les associations n'échappent pas à cette règle. En effet, une stratégie marketing est indispensable aux associations cherchant, de plus en plus, l'adaptation des services rendus aux besoins des bénéficiaires, la sauvegarde des partenaires, l'accroissement du nombre de donateurs et la survie face à l'explosion du tissu associatif.

Cet article a pour objectifs de présenter les résultats d'une recherche exploratoire sur l'application du marketing au niveau des associations marocaines de la province de Ouarzazate, de dresser un état des lieux à ce sujet et de participer à l'amélioration des pratiques existantes tout en dégageant la voie pour une démarche marketing adaptée.

L'étude menée s'est basée sur une approche caractérisée par une analyse descriptive de l'existant sur la base d'un questionnaire. En effet, bien qu'il s'agisse d'une enquête quantitative, elle est complétée par une démarche qualitative en se référant à la technique d'entretien semi directif avec différents responsables au niveau des bureaux exécutifs de ces associations.

L'analyse des données recueillies montre que malgré l'absence d'une approche formalisée du marketing au sein de ces associations, ses pratiques sont en constante amélioration et que l'application de celui-ci dépend souvent des ressources humaines et financières dont ces associations disposent.

Mots clés : Marketing; Associations; Économie sociale et solidaire. 


\title{
Associations and Marketing: An Exploratory Study at the Level of Moroccan Associations in the Province of Ouarzazate
}

\author{
El Kharraz Ouail, PH
}

Faculté des Sciences Juridiques, Economiques et Sociales - Tanger de l'Université Abdelmalek Essaâdi, Maroc

Cherradi Laila, $P A$

Faculté Polydisciplinaire - Ouarzazate de l’Université Ibn Zohr, Maroc

\begin{abstract}
Today, marketing is a key factor for the success of any organization. Associations are not excluded from this rule. Indeed, a marketing strategy is essential for associations which are increasingly seeking to adapt the services provided for the needs of beneficiaries, to safeguard partners, to increase the number of donors and to survive in an environment characterized by an explosion of the associative networks.

This article aims to present the findings of an exploratory research on the application of marketing conducted at the level of some Moroccan associations in the province of Ouarzazate, to draw up an overview of the situation on this subject and to take part in the improvement of existing practices by providing solutions for an adapted marketing approach.

The study was based on an approach characterized by a descriptive analysis of the existing situation on the basis of a questionnaire. In fact, although this is a quantitative survey, we have completed it with a qualitative approach by referring to the semi-directional interview technique with various officials at the level of the executive offices of these associations.

Analysis of the data collected shows that despite the absence of a formal marketing approach within these associations, its practices are constantly improving and that its application often depends on the human and financial resources available to these associations.
\end{abstract}

Keyworlds: Marketing; Associations; Social and solidarity economy.

\section{Introduction}

Les associations sont des organisations spécifiques en raison de leurs objectifs non financiers et de la diversité du public auxquels elles s'adressent. 
Elles peuvent collaborer et concurrencer et attirent l'attention du public positivement et négativement (Gallagher \& Weinberg, 1991).

Il s'agit d'entités démocratiques, portant des projets, composées de membres à la fois utilisateurs et décideurs réunis autour de valeurs et possédant une double hiérarchie salariale et politique (Huet \& Simon, 2007).

Par ailleurs, de plus, et dans un contexte de plus en plus exigeant, les associations doivent non seulement s'adapter et résister à la concurrence mais aussi conserver leurs spécificités tout en innovant. Les associations sont aujourd'hui confrontées à de nombreux défis qui les inquiètent et les laissent démunies. Elles peinent à adapter dans ce contexte leurs modes de contribution à l'intérêt général et à trouver un mode de fonctionnement approprié (La Fonda, 2012).

De plus, il paraît essentiel que, pour accroître leurs capacités financières, attirer plus d'adhérents, mobiliser plus de donateurs, inciter les pouvoirs publics à offrir plus de subventions et adapter mieux les services rendus aux besoins des personnes ciblées, les associations doivent intégrer une stratégie marketing tout en respectant leurs valeurs, leurs objectifs et leur environnement.

Au niveau du Maroc, le rôle des associations occupent une place très importante dans les discours et les décisions des différents acteurs publics et privés surtout en raison de certaines politiques publiques (désengagements, privatisations, ...) et de quelques phénomènes sociaux et culturels (printemps arabe, mondialisation...).

Les associations interviewées de la province de Ouarzazate font face à une concurrence croissante pour solliciter des donateurs (comme la plupart des associations du Maroc) et confrontent plusieurs contraintes spécifiques liées à leur milieu (zone géographique étendue, climat rigide, la non connectivité de la région et la quasi-absence d'entreprises).

A ce stade, il paraît nécessaire de présenter quelques informations relatives à la province de Ouarzazate, sujet de notre enquête. En effet, la province est située au sud-est du Maroc, d'une superficie de $19464 \mathrm{~km}^{2}$, de 297502 habitants majoritairement ruraux (Recensement général de la population et de l'habitat en 2014) et subdivisée en 17 communes dont 2 municipalités: Ouarzazate et Taznakht.

Notre étude s'intéresse surtout à la manière dont les associations de cette province appliquent le marketing ainsi que l'effet de cette démarche sur leurs potentiels à élaborer des programmes efficaces et à sortir de l'enfermement local en améliorant la gestion de leurs activités et en créant des partenariats privilégiés.

Il faut souligner aussi, que l'enquête menée sur l'application du marketing au sein des associations de la province de Ouarzazate, à travers des interviews 
directes avec plusieurs acteurs et responsables, révèle d'importantes pratiques dans ce domaine.

Reste à signaler que notre réflexion portera sur le traitement et l'analyse des trois éléments suivants:

- $\quad$ Les associations et le marketing : Cadre théorique.

- Contexte et méthodologie de recherche.

- Résultats et recommandations.

\section{Les associations et le marketing : Cadre théorique}

L'association est devenue avec ses spécificités et ses particularités l'un des piliers majeurs de l'économie sociale et solidaire. De plus, il devient nécessaire qu'elle utilise des outils et des techniques capables d'améliorer son fonctionnement. A cet effet, certains auteurs croient que le marketing jouera un rôle primordial à ce sujet alors que d'autres ne le pensent pas.

\section{A- L'association : Caractéristiques et spécificités}

Pour comprendre notre question centrale de recherche, il est indispensable de commencer par la présentation de certaines caractéristiques et spécificités des associations :

- Les associations sont des organisations de droit privé au même titre que les entreprises classiques. Mais, contrairement à ces dernières, elles ont un but non lucratif, c'est-à-dire que les porteurs du projet associatif ne doivent pas voir leur patrimoine croître du fait de leurs fonctions dans ces structures (Meier \& Schier, 2008).

- Trois composantes caractérisent le projet associatif à savoir la mission de l'association (qui renseigne sur : son histoire, l'essence de son activité, ses obligations envers son environnement et ses partenaires, ainsi que le rôle qu'elle entend jouer dans la société), les principes de son fonctionnement (qui guident l'organisation dans la fixation de ses objectifs et les actions permettant de les atteindre) et les objectifs durables envers les parties prenantes internes ou externes (Mayaux \& Revat, 1993).

- L'évaluation de l'efficacité de la gouvernance associative demeure, en général, complexe et défaillante du fait de l'absence d'indicateurs spécifiques et synthétiques de mesure de la performance (Bayle, 2001).

- Les sources de financement des associations peuvent être présentées comme suit : la première source est offerte par les cotisations des membres de 1'association. La seconde est composée des réponses aux appels d'offres (lancés par des fondations, des communautés, des gouvernements) : ce sont des financements sur projets. La troisième est proposée par des membres exceptionnels qui font des donations ou des legs. La vente des services constitue une quatrième source de financement (Chauvière et al., 2009). 
- L'association est dans l'obligation de justifier son activité si elle veut obtenir des fonds. Il est donc nécessaire pour elle de disposer d'un système d'information interne capable de lui offrir cette possibilité de justification (Sousi, 2011).

- Les bénévoles s'orientent vers les associations avec des volontés de se former, se professionnaliser ou réaliser une tâche d'exécution qui demandera moins de présence dans le temps (Nicourd, 2009). Ils s'engagent pour participer aux règles collectives, aux pratiques habituelles, aux rituels qui organisent le temps, les tâches et les interactions. Ils sont mobilisés plus pour le collectif que pour eux-mêmes (Sawicki \& Siméant, 2009).

- L'engagement associatif apparaît de plus en plus fréquemment lié à une prise de responsabilité importante au sein des groupements et à une affirmation de compétences spécifiques désormais officiellement reconnues par la validation des acquis de l'expérience au même titre qu'une activité professionnelle (Hély, 2009).

- Les associations étant par nature une structure particulière, composée à la fois de salariés et de bénévoles, la question de la professionnalisation des associations passe nécessairement par une acquisition de compétences organisationnelles (Yolande, 2015).

- Les valeurs de désintéressement, d'engagement citoyen et de générosité constituent le terreau traditionnel des pratiques associatives. Ces normes, intrinsèques au monde associatif, expliquent que le différentiel de rémunération parmi les travailleurs de «l'économie sociale et solidaire » soit très largement accepté (Hély, 2010).

\section{B- L'association : Élément essentiel de l'économie sociale et solidaire}

L'économie sociale et solidaire regroupe une variété d'organisations et d'entreprises qui partagent toutes des objectifs, des valeurs et des principes de fonctionnement sociaux et économiques. Elle désigne des organisations et des entreprises fondées sur des principes de solidarité et de participation et qui produisent des biens et des services tout en réalisant des objectifs à la fois économiques et sociaux (Fonteneau et al., 2010).

Elle se constitue de l'ensemble des coopératives, mutuelles, associations et fondations partageant des particularités qui les distinguent des entreprises individuelles, des entreprises publiques et des sociétés de capitaux (Collette \& Pigé, 2008).

Il est donc nécessaire d'identifier quelques particularités de cette économie à travers la présentation d'une comparaison des spécificités de ses principales composantes.

En effet, le tableau 1 (Defourny \& Develtere, 2009) ci-dessous montre bien les caractéristiques des trois parties essentielles de cette économie à savoir les associations, les coopératives et les mutuelles : 


\begin{tabular}{|c|c|c|c|}
\hline & Association & Mutuelle & Coopérative \\
\hline Rôle & $\begin{array}{l}\text { Fournit des services à } \\
\text { ses membres et / ou à } \\
\text { une communauté plus } \\
\text { large }\end{array}$ & $\begin{array}{l}\text { Fournit des services à } \\
\text { ses membres et / ou à } \\
\text { une communauté plus } \\
\text { large }\end{array}$ & $\begin{array}{l}\text { Fournit des biens et des } \\
\text { services à ses } \\
\text { membres et, dans } \\
\text { certaines circonstances, à } \\
\text { l'ensemble de la } \\
\text { communauté }\end{array}$ \\
\hline $\begin{array}{c}\text { Types de } \\
\text { produits et de } \\
\text { bénéfices }\end{array}$ & $\begin{array}{l}\text { De manière générale, } \\
\text { des biens et des } \\
\text { services res non } \\
\text { marchands, mais } \\
\text { également, de plus en } \\
\text { plus fréquents, du } \\
\text { type marchand. } \\
\text { Selon les méthodes } \\
\text { de mise en œuvre, qui } \\
\text { varient beaucoup, les } \\
\text { membres et la } \\
\text { communauté peuvent } \\
\text { tirer parti des biens et } \\
\text { des services. }\end{array}$ & $\begin{array}{l}\text { Services } \\
\text { essentiellement non } \\
\text { marchands. } \\
\text { Les membres } \\
\text { bénéficient de ces } \\
\text { services en fonction de } \\
\text { leurs besoins. }\end{array}$ & $\begin{array}{lr}\text { Biens et } & \text { services } \\
\text { marchands. } & \\
\text { Chaque membre } \\
\text { bénéficie } \\
\text { ces biens et services } \\
\text { proportionnellement au } \\
\text { nombre de transactions } \\
\text { qu'il ou elle effectue avec } \\
\text { la coopérative (par } \\
\text { exemple, une prime pour } \\
\text { les membres utilisant les } \\
\text { services). }\end{array}$ \\
\hline Adhésion & $\begin{array}{l}\text { Particuliers ou } \\
\text { personnes morales }\end{array}$ & Particuliers uniquement & $\begin{array}{l}\text { Particuliers ou personnes } \\
\text { morales }\end{array}$ \\
\hline $\begin{array}{l}\text { Division de } \\
\text { pouvoir }\end{array}$ & $\begin{array}{l}\text { Le principe "une } \\
\text { personne, une voix" } \\
\text { est appliqué à } \\
\text { l'assemblée générale. }\end{array}$ & $\begin{array}{l}\text { Le principe "une } \\
\text { personne, une voix" est } \\
\text { appliqué lors des } \\
\text { assemblées générales } \\
\text { des membres. }\end{array}$ & $\begin{array}{l}\text { Le principe "une } \\
\text { personne, une voix" est } \\
\text { appliqué lors des } \\
\text { assemblées générales des } \\
\text { membres. }\end{array}$ \\
\hline Financement & $\begin{array}{l}\text { Cotisations et } / \text { ou } \\
\text { dons. Lorsque les } \\
\text { membres } \\
\text { démissionnent, les } \\
\text { cotisations ne sont } \\
\text { pas remboursées. }\end{array}$ & $\begin{array}{l}\text { Cotisations payées à des } \\
\text { intervalles réguliers. } \\
\text { Lorsque les membres } \\
\text { démissionnent, les } \\
\text { cotisations ne sont pas } \\
\text { remboursées. }\end{array}$ & $\begin{array}{l}\text { Souscriptions à des parts } \\
\text { de capital et / ou } \\
\text { d'apports effectués à des } \\
\text { intervalles réguliers. } \\
\text { Lorsque les membres } \\
\text { démissionnent, ils } \\
\text { récupèrent r leur } \\
\text { contribution financière. }\end{array}$ \\
\hline $\begin{array}{l}\text { Distribution de } \\
\text { surplus }\end{array}$ & $\begin{array}{l}\text { Jamais distribué aux } \\
\text { membres. } \\
\text { Doit être réinvesti de } \\
\text { manière socialement } \\
\text { utile. }\end{array}$ &  & $\begin{array}{l}\text { Peut servir de fonds de } \\
\text { réserve pour améliorer } \\
\text { les services ou } \\
\text { développer les activités } \\
\text { de la coopérative. }\end{array}$ \\
\hline
\end{tabular}

Tableau 1. Les principaux mécanismes de fonctionnement de l'économie sociale (Defourny \& Develtere, 2009) 


\section{C- L'association et le marketing : Quelle relation ?}

Pour qu'un produit d'une organisation à but non lucratif survive et se développe, il est nécessaire qu'il soit capable de prendre en compte les besoins sociaux, émotionnels, politiques et intellectuels de ses bénéficiaires (Kotler \& Andreasen, 1991).

Selon Di Sciullo (1988), l'association doit savoir " se vendre ", auprès d'un multi-public composé des adhérents ou usagers potentiels, des pouvoirs publics (agréments, reconnaissances, demandes de subventions) et des sponsors ou donateurs. C'est pourquoi, elle a besoin de connaître des techniques adaptées à la spécificité de leur activité et à la mentalité de son public.

A ce niveau le marketing jouera un rôle très important du moment qu'il permettra à n'importe quelle association de mieux piloter les échanges avec ses différentes parties prenantes dans une situation de concurrence (Mayaux, 2009):

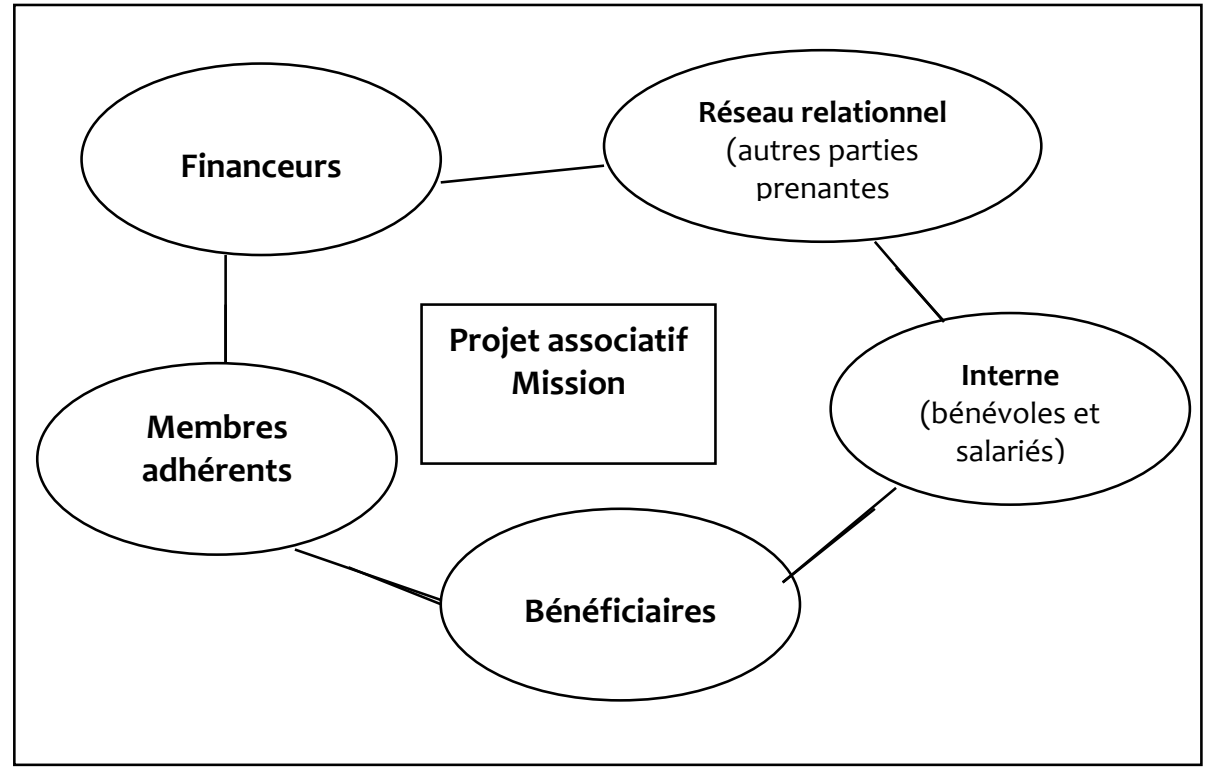

Figure 1. Le marketing associatif, une démarche multipolaire (Mayaux, 2009)

Ainsi, on peut dire que les techniques de marketing permettent à l'association (Mertens, 2009) :

- d'énoncer sa mission, à mieux comprendre les besoins et attentes de ses différents interlocuteurs et à décrire ses propositions à l'échange ;

- d'identifier la plus-value à apporter, communiquer adéquatement sur sa différence et assurer sa pérennité ;

- de créer une valeur d'échange pour tout son public (financeurs, bénévoles, salariés, famille des usagers, médias) et non seulement aux usagers. 
A ce niveau Gallopel-Morvan et al. (2013) ont définis une démarche marketing spécifique aux associations présentée dans le schéma suivant :

-Ressources de l'association: collecter des fonds, motiver et fidéliser les bénévoles -Faire connaître l'association à ses publics -Agir/mission: évoluer les attitudes, adopter un nouveau comportement

1 - Définition des objectifs de l'association (en fonction de son identité et de sa mission)

« Le marketing mix » (les 4 P) c'est définir :

- Service proposé

- Son prix

- Sa place (la manière dont il est distribué)

- Sa promotion (la communication)

Figure 2. La démarche marketing pour une association (Gallopel-Morvan et al., 2013)

Cependant, d'autres auteurs pensent que le marketing est inadapté à l'activité associative. Dacheux (1998), par exemple, avance à ce niveau plusieurs raisons :

*La démarche marketing est liée au marché et si celui-ci n'existe pas (comme pour le cas des associations), il sera totalement insensé de parler de marketing associatif.

*Segmenter revient à identifier des bons et des mauvaises cibles. Cette action de discrimination est contraire à l'esprit associatif.

*Certaines techniques marketing auront un impact négatif sur l'image de l'association qui les appliquent du moment que celle-ci sera amenée à délaisser quelques valeurs en contrepartie de ressources financières faibles.

* La recherche pour de nouvelles alternatives, mènera les associations à un recours aux conseillers spécialisés, ce qui conduira à une application de marketing sans autant prendre en compte les spécificités du secteur associatif.

Reste à signaler à ce niveau, que le but de ce papier n'est pas de débattre de la légitimité de l'utilisation ou non du marketing aux associations ou encore de prendre position mais plutôt d'essayer de présenter les avis sur le sujet et surtout d'explorer sur l'application du marketing au niveau de quelques associations marocaines.

\section{Etude empirique : Contexte et méthodologie de recherche A- Contexte de l'étude}

Les associations marocaines rencontrent aujourd'hui, dans leurs parcours d'essor et de transition plusieurs défis d'ordre général et d'autres en relation direct avec le milieu marocain. 
a- Défis d'ordre général : Il s'agit ici de défis d'ordre général dont l'association doit faire face. A ce niveau, on peut citer les exemples suivants:

- Dans une période où l'instabilité devient la règle et la stabilité est l'exception, il est essentiel au tissu associatif d'ajuster ses buts afin de contribuer aux objectifs de développement de l'économie.

- L'augmentation du nombre des organisations associatives ne peut à elle seule servir comme solution pour lutter contre les effets du chômage, l'exclusion des catégories sociales fragiles et la pauvreté. Il est nécessaire de miser sur la qualité que sur la quantité des services rendus.

- Les composants de la société en général portent plus d'attention aux nouvelles technologies et aux réseaux sociaux. D'où l'importance de tirer profit de ces outils et applications dans l'organisation et la gestion des associations.

- Les associations constituent l'un des piliers de la vie démocratique, sociale et culturelle. Elles interviennent dans de multiples domaines et participent activement à la mise en œuvre des politiques publiques. Elles constituent ainsi des espaces de confiance, d'éducation citoyenne, de solidarité et d'autonomie, tout en apportant des réponses à des situations à travers des projets d'intérêt général et des activités sans finalité lucrative (MAFC, 2015).

b- Défis relatifs au Maroc : La dynamique connue par le tissu associatif au Maroc ne peut nuancer l'existence des obstacles :

* Tout d'abord, les conditions pour un partenariat égalitaire Etat/associations ne sont pas encore remplies, en raison d'un manque de transparence caractérisant le processus d'appel à projets.

* Les fonds propres des associations marocaines sont très limités.

* Un développement insuffisant au niveau du bénévolat, la principale richesse humaine des associations.

* L'enveloppe budgétaire dédiée à la promotion économique de l'économie sociale et solidaire reste très limité (Cour des Comptes, 2018).

\section{B- Méthodologie de l'étude}

L'étude empirique a été réalisée sur la base d'un questionnaire guide comportant à la fois des questions fermées et ouvertes concernant plusieurs axes de la démarche marketing au niveau des associations à savoir : segmentation, ciblage, positionnement, etc.

Ainsi, et pour pouvoir répondre à nos interrogations, il est nécessaire d'établir une liste des associations au niveau de la province de Ouarzazate objet de notre enquête. Celle-ci a touché différents types d'associations. De plus, sur un total de 56 associations localisées sur le terrain, 48 ont répondues à notre questionnaire : 


\begin{tabular}{|c|c|c|c|}
\hline $\begin{array}{l}\text { 1. Tissu associatif de } \\
\text { Ouarzazate pour le } \\
\text { développement } \\
\text { (TAOD) }\end{array}$ & $\begin{array}{lr}\text { 13. } & \text { Association } \\
\text { sportive } & \text { kanadil } \\
\text { Idlssane } & \end{array}$ & $\begin{array}{l}\text { 25. Association } \\
\text { Annahda }\end{array}$ & $\begin{array}{lr}\text { 37. Tigmi rakimte } \\
\text { pour } & \text { le } \\
\text { développement social } \\
\text { et le tourisme culturel } \\
\end{array}$ \\
\hline $\begin{array}{l}\text { 2. Association Oasis } \\
\text { Verte des femmes et } \\
\text { des enfants pour le } \\
\text { développement et la } \\
\text { solidarité }\end{array}$ & $\begin{array}{l}\text { 14. Association } \\
\text { Taznakhte du } \\
\text { Tourisme solidaire et } \\
\text { Envronnement }\end{array}$ & $\begin{array}{l}\text { 26. Association Sud } \\
\text { contre le Sida section } \\
\text { Ouarzazate }\end{array}$ & $\begin{array}{lr}\text { 38. } & \text { Association } \\
\text { Basma des } & \text { enfants } \\
\text { abandonnés } & \end{array}$ \\
\hline $\begin{array}{lr}\text { 3. Association } & \text { des } \\
\text { Echos pour ra } \\
\text { solidarité et } & \text { le } \\
\text { développement } & \\
\text { Ouarzazate (AESD) } \\
\end{array}$ & $\begin{array}{l}\text { 15. Association } \\
\text { sportive sidi daoud }\end{array}$ & $\begin{array}{lrr}27 . & \text { Association } \\
\text { Oxygène } & \text { pour } & \text { la } \\
\text { promotion } & \text { de } & \text { la } \\
\text { femme } & & \end{array}$ & $\begin{array}{l}\text { 39. Association } \\
\text { Auberge de jeunes de } \\
\text { Ouarzazate }\end{array}$ \\
\hline $\begin{array}{l}\text { 4. Association Tichka } \\
\text { pour le développement } \\
\text { et la coopération }\end{array}$ & $\begin{array}{l}\text { 16. Association de } \\
\text { soutien de l'école } \\
\text { Najah au lycée } \\
\text { technique sidi Daoud }\end{array}$ & $\begin{array}{l}28 . \\
\text { Yahya }\end{array}$ & $\begin{array}{l}\text { 40. Association Borj } \\
\text { Almahaba }\end{array}$ \\
\hline $\begin{array}{l}\text { 5. Association de } \\
\text { développement } \\
\text { d'avenir à Ouarzazate }\end{array}$ & $\begin{array}{l}\text { 17. Association } \\
\text { marocaine des droits } \\
\text { humains }\end{array}$ & $\begin{array}{l}29 . \quad \text { Association } \\
\text { Apicole Azoukany }\end{array}$ & $\begin{array}{l}\text { 41. Association Bnate } \\
\text { Zaouya de aourirt }\end{array}$ \\
\hline $\begin{array}{l}\text { 6. Association } \\
\text { Tassaounte pour le } \\
\text { développement }\end{array}$ & $\begin{array}{ll}\text { 18. Association } & \mathrm{Al} \\
\text { Maarifa pour } & \text { le } \\
\text { développement } & \\
(\mathrm{AMDT}) & \end{array}$ & $\begin{array}{lr}\text { 30. } & \text { Espace } \\
\text { Association } & \text { Todra El } \\
\text { oulia } & \\
\end{array}$ & $\begin{array}{l}\text { 42. Club Ouarzazate } \\
\text { pour Média et Presse }\end{array}$ \\
\hline $\begin{array}{l}\text { 7. Association Al } \\
\text { Amal De Cancer } \\
\text { Ouarzazate (aaco) }\end{array}$ & $\begin{array}{l}\text { 19. Association Alwan } \\
\text { de peinture et de l'art }\end{array}$ & $\begin{array}{lr}\text { 31. } & \text { Association } \\
\text { Alkarama pour le } \\
\text { développement des } \\
\text { handicapés }\end{array}$ & $\begin{array}{l}\text { 43. Association } \\
\text { Errahma pour les } \\
\text { mères des orphelins }\end{array}$ \\
\hline $\begin{array}{l}\text { 8. Association Toudert } \\
\text { pour le développement } \\
\text { de la femme }\end{array}$ & $\begin{array}{l}\text { 20. Association Draa } \\
\text { des diabétiques }\end{array}$ & $\begin{array}{l}\text { 32. Club sportif Amal } \\
\text { Ait Kedif }\end{array}$ & $\begin{array}{lr}44 . & \text { Association } \\
\text { Tinouar } & \text { de } \\
\text { développement } & \text { et } \\
\text { solidarité } & \\
\end{array}$ \\
\hline $\begin{array}{l}\text { 9. Association basmat } \\
\text { Khayr pour le } \\
\text { développement } \\
\text { durable (ABKDD) } \\
\end{array}$ & $\begin{array}{ll}\text { 21. } & \text { Association } \\
\text { Mawadda pour le } \\
\text { développement et la } \\
\text { solidarité }\end{array}$ & $\begin{array}{l}\text { 33. Club Annasr du } \\
\text { Sport et Dulture Ait } \\
\text { Kedif }\end{array}$ & $\begin{array}{l}\text { 45. Association Union } \\
\text { des femmes pour le } \\
\text { développement }\end{array}$ \\
\hline $\begin{array}{l}\text { 10. Association des } \\
\text { jeunes fedragoume } \\
\text { pour la culture et le } \\
\text { développement }\end{array}$ & $\begin{array}{lr}\mathbf{2 2} & \text { Association } \\
\text { provinciale } & \text { des } \\
\text { éleveurs d'abeille } \\
\text { (Chems ouarzazate) }\end{array}$ & $\begin{array}{l}\text { Associatif de } \\
\text { ate pour le } \\
\text { pement }\end{array}$ & $\begin{array}{l}\text { 46. Association les } \\
\text { énergies de la jeunesse }\end{array}$ \\
\hline $\begin{array}{lr}\text { 11. } & \text { association } \\
\text { Anmougar } & \text { Tizgui } \\
\text { N'Lilaine } & \end{array}$ & \begin{tabular}{lr} 
23. & \multicolumn{2}{c}{ Association } \\
Izougad pour & le \\
développement & et \\
l'environnement & \\
\end{tabular} & $\begin{array}{l}\text { 35. Association Al } \\
\text { Wafa des Diabétiques } \\
\text { de la province de } \\
\text { Ouarzazate } \\
\end{array}$ & $\begin{array}{l}\text { 47. Association } \\
\text { Tarmigt pour l'enfant } \\
\text { handicapé }\end{array}$ \\
\hline \begin{tabular}{lrr}
12. & \multicolumn{2}{r}{ Association } \\
d'amitié pour & les \\
utilisateurs des & eaux \\
souterrains à des & fins \\
agricoles et & la \\
conservation & de \\
l'environnement et le \\
développement & \\
\end{tabular} & $\begin{array}{l}\text { 24. Association de } \\
\text { développement et de } \\
\text { solidarité pour les } \\
\text { propriétaires te les } \\
\text { chauffeurs de petits } \\
\text { taxis }\end{array}$ & $\begin{array}{l}\text { 36. Association des } \\
\text { guides touristiques } \\
\text { attachés à la Kasbah } \\
\text { (AGTAKTO) }\end{array}$ & $\begin{array}{l}\text { 48. Association de } \\
\text { bienfaisance } \\
\text { Islamique (Dar Talib) }\end{array}$ \\
\hline
\end{tabular}

Tableau 2. Liste des associations interviewées 
Les réponses ont été obtenues grâce aux questionnaires distribués aux différents responsables (présidents, secrétaires généraux, trésoriers) au sein des associations interrogées durant l'année 2018. En effet, bien qu'il s'agisse d'une enquête quantitative, elle est complétée par une démarche qualitative à travers des entretiens semi-directifs afin de recueillir d'avantage d'informations et pour éclaircir certaines réponses.

L'étude a permis de tirer des exemples, des résultats et des conclusions qui sont très importantes dans le domaine du marketing associatif.

\section{Etude empirique : Résultats et recommandations}

\section{A- Résultats de l'étude}

La présentation des résultats et des conclusions de l'étude constitue un élément très important dans le processus d'analyse de l'application du marketing au niveau des associations interrogées.

a- Présentation des résultats : L'étude minutieuse de l'ensemble des réponses tirées des questionnaires remplis par les associations constituant notre échantillon de recherche révèle des résultats très intéressants :

* Au niveau de la discipline : L'application du marketing en tant que discipline n'est pas très développée au sein des associations de Ouarzazate. Les résultats suivants le montrent très clairement :

- $24 \%$ des associations interrogées possèdent une équipe dédiée aux pratiques de marketing au sein de leur structure organisationnelle. Le reste des associations a justifié son absence essentiellement en raison de son attachement à une autre équipe et à cause de leurs petites tailles.

- Les principaux objectifs recherchés à travers l'application du marketing au niveau des associations interviewées restent successivement : la motivation et la fidélisation des bénévoles, la collecte des fonds et l'amélioration de l'image et l'identité de l'association :

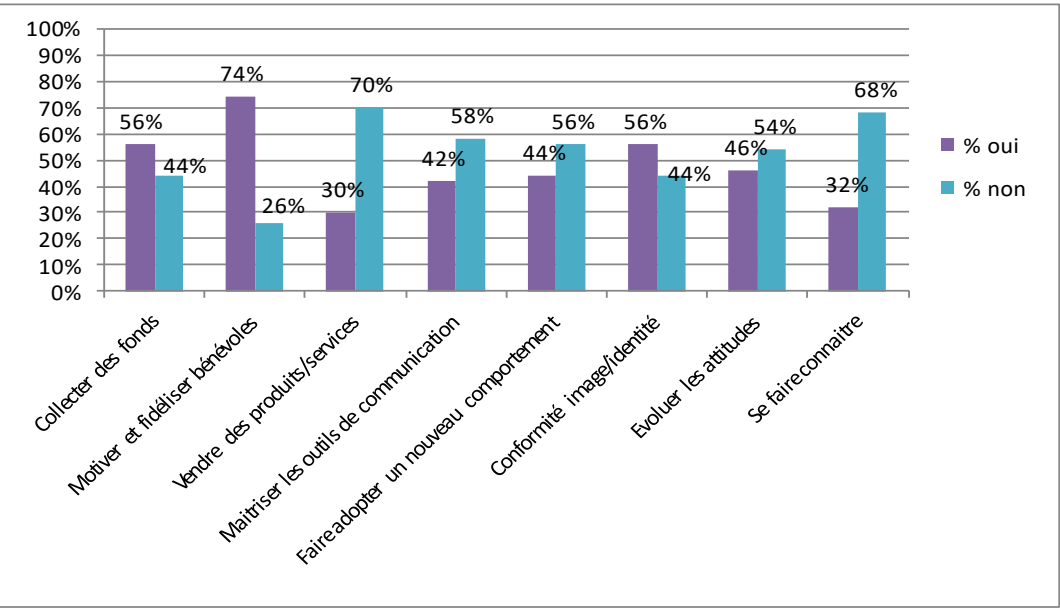

Figure 3. Les objectifs marketing recherchés par les associations interviewées 
- 34\% des associations déclarent utiliser au moins l'une des trois techniques documentaire, quantitative et qualitative pour réaliser une étude de marché.

- 74\% des associations interrogées possèdent une base de données actualisée de leur public-cible comportant leurs comportements, attitudes et besoins.

$-72 \%$ de ces mêmes associations réalisent une analyse de leur environnement.

* Concernant la communication : La communication est un outil primordial pour les associations. A ce niveau, les résultats se présentent comme suit :

- $88 \%$ des associations considèrent la communication comme fondamentale pour leur continuité et pour faire connaitre le projet associatif.

$-72 \%$ des associations utilisent surtout une communication persuasive et $68 \%$ pensent que les messages qu'elles passent sont souvent adaptés au public ciblé.

- 48\% des associations manquent de professionnels en communication au sein de leurs structures et le reste dispose d'une équipe pour garantir un flux d'informations entre les différents partenaires de l'association.

- Comme moyen de communication, l'enquête a révélé aussi une fréquente utilisation, par les associations, de l'internet et des relations publiques, suivit respectivement du phoning et campagnes e-mailing puis l'affichage, une utilisation modérée de la radio et de la presse. La télévision, les salons et les foires sont faiblement utilisés :

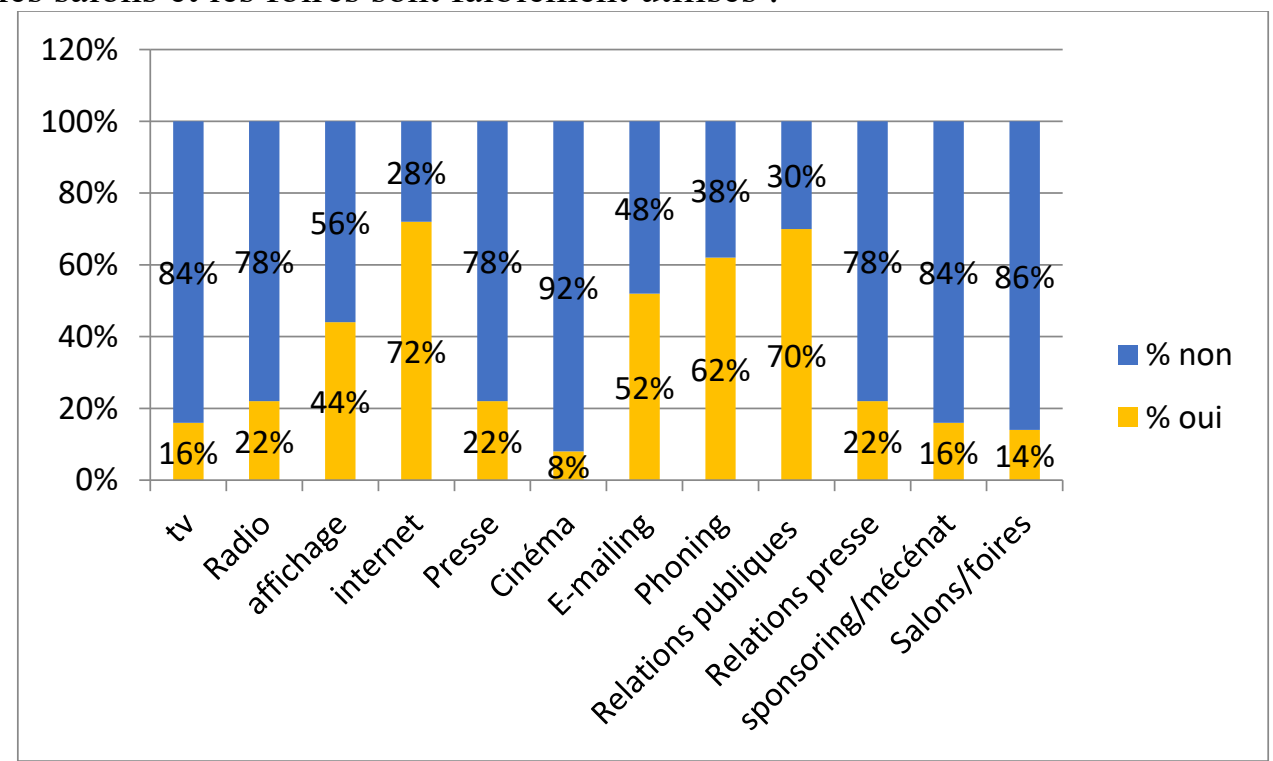

Figure 4. Vecteurs de communication utilisés par les associations interrogées 
- D'autres moyens de communication sont employés par les associations comme les compagnes médicales, les visites sensationnelles, les débats, les congrès, les conférences et les spectacles.

* Pour la collecte des fonds et le bénévolat : Généralement, les associations sont très intéressées par ces deux éléments. Les résultats trouvés à ce niveau sont les suivants :

- En amont de chaque opération de collecte des fonds, l'enquête a montré que les associations essayent de redéfinir leurs objectifs de collecte, de composer leurs équipes de bénévoles, de préparer leurs argumentaires de la compagne et de déterminer les donateurs potentiels.

- Les outils de collecte des fonds fréquemment utilisés par les associations sont respectivement : les événements, le mailing de collecte et la collecte sur internet.

- 80\% des associations rencontrent des difficultés à mobiliser des bénévoles. Les raisons évoquées pour expliquer cela sont respectivement le manque de motivations des bénévoles, l'emploi du temps chargé des candidats et le manque du temps des membres des associations pour les accompagner et les former.

- La démotivation des bénévoles résulte surtout du manque de soutien et de la non valorisation du travail réalisé.

* Concernant la segmentation du public-cible : Les résultats de l'enquête concernant la segmentation du public-cible se présentent ainsi :

- 78\% des associations confirment appliquer la segmentation alors que le reste des associations identifient la province de Ouarzazate et son entourage comme cible.

- Pour les critères de segmentation fréquemment utilisés par les associations interviewées, l'enquête montre qu'elles favorisent nettement les critères traditionnels: géographiques, sociodémographiques et socioéconomiques.

- $62 \%$ des associations déclarent que leurs services s'adressent à des besoins connus de la population cible de l'association, tandis que $38 \%$ ont répondues que leurs services ne s'adaptent «pas toujours » aux besoins du public.

* Autres résultats : Les résultats pour certains d'autres aspects du marketing associatif peuvent être présentés comme suit :

- $84 \%$ des associations procèdent à l'évaluation des actions sociales.

- A la fin de chaque action associative, une réflexion est déclenchée par les associations afin de redéfinir les objectifs, de contrôler l'efficacité des actions et d'étudier le public visé.

- Un faible intérêt est accordé à l'évaluation et à l'analyse du temps consacré au déroulement de l'action associative. 
marketing.

- $14 \%$ des associations confondent la communication avec le

- $88 \%$ des associations déclarent réaliser une étude pour définir les comportements, les attitudes et les besoins de leur public.

- L'entretien avec les responsables montre que les facteurs influençant l'application du marketing social sont respectivement : faible niveau cognitif, faible engagement des membres, manque de cadres compétents et de spécialistes, faible soutien public en termes de financement et d'encadrement, manque de motivation et manque de formation.

b- Les conclusions de l'étude : Les conclusions à tirer de cette enquête sont multiples, mais les plus importantes, à nos yeux, se présentent comme suit :

- Même si, en tant que discipline, le marketing reste peu développé au sein des associations et certaines de ses pratiques sont en amélioration.

- Le tissu associatif est enfermé dans un cycle vicieux : insuffisance des moyens, des actions limitées, manque de fonds, faible plan d'actions, démotivations des bénévoles. Ce qui rend l'application du marketing très difficile.

- Les associations reconnaissent l'importance d'un véritable système de recueil et d'analyse des données de leur environnement pour saisir les opportunités et augmenter leur capacité à mieux planifier les actions.

- La segmentation du public-cible reste la plupart du temps mal élaborée ce qui implique souvent l'inadaptation des services rendus aux besoins du publics (bénéficiaires excluent de l'action ; un public non concerné par l'action) ou des retombées très faibles des actions.

- Les associations préfèrent une communication persuasive pour influencer les attitudes, les opinions et le comportement des interlocuteurs cibles. A ce sujet, elles communiquent avec presque deux catégories d'audiences à savoir les donateurs et les bénéficiaires avec un message qui varie entre un contenu généralisé ou ciblé.

- Le marketing au niveau des associations dépend des ressources humaines et financières dont celles-ci disposent.

\section{B- Recommandations de développement}

Parmi les recommandations qui peuvent être proposées pour l'amélioration de l'application du marketing associatif, on peut citer les éléments suivants :

- Les associations doivent se focaliser sur l'encouragement d'une culture favorable à une démarche marketing. Il s'agit d'accentuer les sentiments des membres et des adhérents sur son importance et de son impact positif sur la croissance de leurs structures et de leurs parcours professionnels. 
- Définir une stratégie de marketing répondant aux besoins des bénévoles et des salariés tout en respectant les valeurs des associations en question.

- Les associations doivent intensifier l'utilisation des nouvelles technologies pour diffuser leurs messages et convaincre leurs audiences cibles (Internet, réseaux sociaux, etc.) avec une formation permanente sur la manipulation de ces instruments.

- L'ouverture des universités avec ses facultés et ses écoles supérieures de proximités aux besoins des associations en matière d'accompagnement et de formation en marketing.

- Améliorer d'avantage les relations avec la presse ne peut que faciliter la diffusion des informations et des messages et aider les associations à une meilleure communication interne et externe.

- Encourager la collaboration en matière de marketing avec d'autres partenaires pour faciliter son utilisation au niveau de ces associations.

- Renforcer le sentiment d'appartenance aux associations pour les différents acteurs en les intégrant dans l'élaboration de différentes pratiques de marketing.

\section{Conclusion}

Les associations sont des organisations non marchandes devant assurer leur développement et celui de leurs produits ou services d'action sociale sur des marchés souvent non solvables mais pris en charge pour le compte de la collectivité par un certain nombre de relais et d'administrations des affaires sociales (Dobiecki, 2007).

Le mouvement associatif est ancré dans la tradition culturelle et sociale marocaine, notamment sous la forme de «jmaa ${ }^{l} »$, de «twiza $a^{2}$ et de corporations professionnelles. Les associations ont ainsi joué un rôle important dans l'organisation, la régulation et la représentation des métiers et des professions de l'agriculture, de l'artisanat, du commerce et de la culture. Elles ont également été très actives dans les domaines de l'éducation civique, de l'action politique pour l'indépendance puis du plaidoyer en faveur de la démocratie et de l'Etat de droit (CESE, 2017).

Sur le terrain, ces associations souffrent de plusieurs contraintes d'ordre économiques, administratives et organisationnelles. De ce fait, il est nécessaire que ces associations s'engagent dans des approches managériales capables de contribuer à surmonter ces différents obstacles. La démarche marketing peut constituer, d'après certains auteurs, un instrument très important dans ce domaine.

\footnotetext{
${ }^{1}$ Appelé aussi « l'assemblée des hommes du village » est une structure qui gère les affaires de la communauté villageoise et au sein de laquelle les principales familles sont représentées. ${ }^{2}$ Le terme tiwiza ou twiza est une structure traditionnelle d'échange généreux par l'activité.
} 
Généralement, une stratégie marketing pour une association se fonde sur trois bases essentielles à savoir le choix du public visé, sa spécificité qui la distingue d'une autre et les différents éléments adéquats du marketing mix. Cette stratégie pourra permettre une concordance totale entre les valeurs et les finalités des associations, ses moyens humains et financiers et les changements incessants au niveau de l'environnement associatif.

Par ailleurs, notre étude a montré que ces associations ont besoins de comprendre l'utilité d'une fonction marketing et d'assimiler sa démarche (outils et étapes-clés) tout en mettant leurs valeurs en action via des produits et des services répondant aux besoins de leurs bénéficiaires. Pour cela, une reconfiguration de la place du marketing dans la structure de ces associations est vitale.

De ce fait, elles doivent investir en permanence dans une réflexion sur la définition des produits et services rendus afin de cerner la diversité de leurs publics, leurs besoins, leurs capacités à apporter une réponse adaptée, les retombées économiques et sociales de leurs actions par rapport aux autres associations et au niveau de leurs domaines d'activité.

De plus, il faut reconnaître que même si la majorité de ces associations ne disposent pas de toutes les ressources humaines, techniques et financières pour concevoir et améliorer l'utilisation du marketing au sein de leurs entités, il reste néanmoins nécessaire au développement de plusieurs aspects de leur fonctionnement. Ce qui leurs manquent le plus à ce niveau, c'est la volonté de l'appliquer et une solide culture pour le réussir. Il reste à noter enfin, que ces deux derniers éléments constituent deux thèmes prometteurs pour l'étude et l'analyse des différents chercheurs et praticiens intéressés par ce domaine.

\section{References :}

1. Bayle, E. (2001). Les modes de gouvernance des organisations à but non-lucratif : une étude appliquée aux fédérations sportives nationales. Revue des sciences de gestion, septembre $\mathrm{N}^{\circ} 188-189$ dossier (« Piloter l’entreprise »), pp. 54-69.

2. Chauvière, M., Hély, M., Pattieu, S., Pierson, M., Rousseau, F., Zunz, O., Chessel, M-E., Nicourd, S. \& Capuano, C. (2009). DÉBAT: L'association, l'entreprise et l'administration : quelle circulation des normes de gestion? ESKA, 2009/3 n 56, pp.131-144.

3. Collette, C. \& Pigé, B. (2008). Economie sociale et solidaire, Gouvernance et contrôle. Édition Dunod, 168 pages.

4. Conseil Economique, Social et Environnemental (CESE). (2017). Statut et dynamisation de la vie associative. Rapport de la commission permanente chargée des Affaires Sociales et de la Solidarité, Maroc.

5. Cour des Comptes. (2018). Rapport d'activités relatif aux exercices 2016 et 2017. Rapport général, Vol. I, Rabat, 767 pages. 
6. Dacheux, É. (1998). Associations et communication: Critique $d u$ marketing. CNRS Éditions, Paris, 160 pages.

7. Defourny, J. \& Develtere, P. (2009). The Social Economy: the Worldwide Making of a Third Sector. In J. Defourny, P. Develtere, B. Fonteneau \& M. Nyssens (Eds.). The Worldwide Making of the Social Economy, Innovations and Changes. Leuven \& The Hague: Acco, pp. 15-40.

8. Di Sciullo, J. (1988). Marketing et Communication des associations. Juris Service, Paris.

9. Dobiecki, B. (2007). Marketing social: Du non-profit au profit collectif. Editions ESKA, n 2, Vol. 7, pp. 91-116.

10. Fonteneau, B., Neamtan, N., Wanyama F., Pereira, M. L. \& Poorter, M. (2010). Social and Solidarity Economy: Building a Common Understanding. International Training Centre of the International Labour Organization, In support of the First edition of the Social and Solidarity Economy Academy, 25-29th October, ITC ILO, Turin, Italy.

11. Gallagher, K. \& Weinberg, C.B. (1991). Coping with success: new challenges for nonprofit marketing. Sloan Management Review, 33(1), pp. 27-42.

12. Gallopel-Morvan, K., Birambeau, P., Larceneux, F. \& Rieunier, S. (2013). Marketing et Communication des Associations - 2e édition. Dunod, Paris.

13. Hély, M. (2009). Présentation. Penser le monde associatif comme un monde du travail. Dans : M. Hély, Les métamorphoses du monde associatif (pp. 1-19), Paris cedex 14, France: Presses Universitaires de France.

14. Hély, M. (2010). Le travail " d'utilité sociale » dans le monde associatif, Management \& Avenir, 2010/10 (n40), pp. 206-217.

15. Huet, J. \& Simon, A. (2007). Pouvoir et légitimité dans les associations, L'Expansion Management Review, vol. 125, n², pp. 68.

16. Kotler, P. \& Andreasen, A. (1991). Strategic Marketing for Nonprofit Organizations. 4th edition, Englewood Cliffs (NJ), Prentice-Hall.

17. La Fonda. (2012). Accompagner les mutations associatives: Etats des lieux et propositions. Disponible sur: https://fonda.asso.fr/ressources/accompagner-les-mutationsassociatives (consulté le 25/04/2019).

18. Mayaux, F. \& Revat, R. (1993). Marketing pour association, l'efficacité au service de vos valeurs. Edition Liaisons, 140 pages. 
19. Mayaux, F. (2009). Le marketing au service des associations : légitimité et spécificités. Entreprises et histoire, vol. 56, no. 3, pp. 98116.

20. Meier, O. \& Schier, G. (2008). Quelles théories et principes d'actions en matière de gouvernance des associations?. Management \& Avenir, 2008/6 (n 20), pp. 179-198.

21. Mertens, S. (2009). Les spécificités du marketing des ASBL, pp 23 -53, Chapitre in Coipel, M., Marée, M. et al. (2009). Asbl Et Activites Marchandes. Edipro, 166 pages.

22. Mouvement Associatif de Franche Comté (MAFC). (2015). Les associations face aux mutations : Subir, Réagir, Agir... ?. Conférence régionale de la vie associative, 26 septembre, Besançon.

23. Nicourd, S. (2009). Éducation populaire : Organisation du travail associatif et action publique. ESKA, 2009/3 n 56, pp.62-72.

24. Sawicki F. \& Siméant J. (2009). Décloisonner la sociologie de l'engagement militant, Note critique sur quelques tendances récentes des travaux français », Sociologie du travail, 51, pp. 97-125.

25. Sousi G. (2011). Le Fonctionnement des associations: étude jurisprudentielle (Droit, économie, gestion). Édition L'Hermès, 157 pages.

26. Yolande, F. (2015). La professionnalisation des associations par les pratiques de gestion des compétences des acteurs associatifs produitelle un processus de changement organisationnel?. Recherches en Sciences de Gestion, 2015/1 ( $\left.{ }^{\circ} 106\right)$, pp. 113-133. 